\title{
In-situ understanding of pore nucleation and growth in polyurethane foams by using real-time synchrotron X-ray tomography
}

\author{
S. Pérez-Tamarit ${ }^{1, *}$, E. Solórzano ${ }^{1}$, R. Mokso $^{2,3}$, M. A. Rodríguez-Pérez ${ }^{1}$. \\ ${ }^{1}$ CellMat Laboratory, Condensed Matter Physics Department, University of Valladolid, \\ Paseo de Belén, 7 47011, Valladolid \\ ${ }^{2}$ Swiss Light Source, Paul Scherrer Institute, Villigen, Switzerland \\ ${ }^{3}$ MAX IV Laboratory, Lund University, Lund, Sweden \\ * Corresponding author saul.perez@fmc.uva.es
}

\begin{abstract}
The nucleation and growth of pores in PU foams have been visualized and quantified for the first time in 4D using in-situ tomographic microscopy. Acquiring sequences of 3D images in $156 \mathrm{~ms}$ each, we describe homogeneous and heterogeneous nucleation and subsequent bubble growth dynamics for materials with and without nucleating nanoparticles. Exploiting the time resolved tomographic sequences we obtained the bubble nucleation rate, bubble density and size evolution, based on which the diffusion coefficient of gas molecules in polymer can be calculated, evidencing the reduction of diffusivity provoked by the inclusion of nanoparticles. We found that nucleation and growth are in a large extend decoupled in time.
\end{abstract}

Keywords: polyurethane; synchrotron tomography; 4D imaging; foaming; porous polymers. 


\section{Introduction}

Bubble nucleation and growth are critical steps in any foaming process. They are linked to a thermodynamic instability in a specific gas-liquid system. Gas is dissolved into the liquid phase until the solubility limit is reached. At this moment, it precipitates in form of sub-micron metastable nuclei that may both collapse or grow upon a critical diameter. As part of this process a thermodynamical energy barrier must be overcome according to the classical homogeneous nucleation theories [1-3]. Moreover, the presence of particles and/or impurities in the liquid phase may significantly alter the homogeneous bubble nucleation of purified media due to further physical interactions. Under this situation, the local perturbations at gas/liquid interface could facilitate the formation of bubble nuclei increasing consequently the total number of nucleated bubbles. This is the so-called heterogeneous nucleation process [4] that typically reduces the nucleation energy barrier yielding superior bubble densities [5]. Furthermore, it is well known that both size and shape of nucleating particles influence their nucleation potential. The higher is the shape irregularity and lower the size of the particles, the higher is the expected nucleation capability [6]. Therefore, nanoparticles seem to be ideal nucleating agents for bubbles [7]. Nevertheless, interfacial compatibility plays also important role. In this sense the most interesting nucleating agents are those with chemically-modified surface, thus enhancing the interaction between additive and matrix [8]. This surface compatibilization may affect apparent viscosity and surface tension among other rheological properties [9]. After nucleation has taken place, bubble growth occurs. The equations governing these interactions depend, among other factors, on the diffusion equations considering the available amount of gas and the kinematic characteristics of the fluid [10, 11]. In addition, degeneration mechanisms of the cellular structure such as film drainage, coarsening or coalescence may occur $[12,13]$.

The fragility of these systems and the complexity of physico-chemically coupled mechanisms need from advanced nondestructive techniques with sufficient temporal and spatial resolution. Our research group has used time-resolved X-ray imaging (so-called radioscopy) to study the bubble nucleation and growth in different polymer/gas systems with considerable success [14-18]. These studies were performed in 2D, at a rather low speed $(2 \mathrm{~Hz})$ and with a considerable time span from mixing to X-ray monitoring of typically 40-50 seconds. 
Last developments at synchrotron facilities, with X-ray intensities $10^{6}$ higher than lab systems, open the possibility of performing time-resolved X-ray tomography experiments reaching up to $20 \mathrm{~Hz}$ micro-tomography ( $\mu$-CT) scans [19-21]. In addition, the coherence of synchrotron radiation enables the use of phase contrast X-ray imaging, which permits enhancing the contrast for low absorbing materials such as polymers [22, 23]. This is a powerful strategy that for instance has allowed to distinguish two polymers with similar characteristics (density and constituent elements) such as polystyrene and polycarbonate [24]. In this study we make use of these recent advances to perform time resolved X-ray tomography experiments analyzing the bubble generation and growth in reactive polyurethane (PU) foams in absence/presence of silica nanoparticles reaching temporal resolutions of $156 \mathrm{~ms}$ per scan with $3 \mathrm{D}$ voxel size of $3.2 \mu \mathrm{m}$. One of the additional innovations included in our study was the design of an in-situ mixing system to eliminate the span time and scan the samples immediately after mixing (see supplementary material for more details about the mixing system and the mixing process).

\section{Experimental}




\subsection{Materials and foam production}

A bi-component PU formulation from BASF was selected for this investigation. Previous research has proved this system is stable, not showing degeneration mechanisms such as coalescence or coarsening [15]. The mixing of two components, isocyanate (IsoPMDI 92140) and polyol blend (Elastopor H 1501/1) (which contains catalyst, surfactants, blowing agent and polyol) promotes simultaneous blowing and gelling reactions that results into a solid cellular polymer after few minutes (gel time $145 \pm 18 \mathrm{~s}$ ) [25]. The nanoparticle selected for the comparative study was a hydrophobic fumed nanosilica (R812 from Evonik Industries, Germany) post-treated with hexamethyldisilazane [26]. $3 \%$ wt of nanosilica was previously dispersed (120s) in the isocyanate component. A miniature specific mixing system was designed and used in order to in-situ promote the chemical reactions. Both PU components were placed inside a $75 \mu \mathrm{m}$ thickness kapton cup $(\mathrm{h}=40 \mathrm{~mm}, \emptyset=12 \mathrm{~mm}$ ) coupled to the tomography rotation table (Aerotech ABRT200). The mixture was stirred during $15 \mathrm{~s}$ at $1500 \mathrm{rpm}$, and then the first tomography of the sequence was acquired. In this study, we have analyzed only the first 90s of the process in which both nucleation and approximately $70 \%$ of the total expansion take place.

\subsection{Real time synchrotron X-ray tomography}

Experiments were performed at the TOMCAT beamline of the Swiss Light Source at the Paul Scherrer Institute (Villigen, Switzerland). The samples were illuminated by $20 \mathrm{keV}$ monochromatic X-ray beam resulting in phase contrast projections recorded with a PCO.Dimax high speed detector coupled to a $100 \mu \mathrm{m}$ thick LuAg:Ce scintillator. The evolution of one sample was typically recorded in over 80 tomographic scans, in each scan 300 angular projections over $180^{\circ}$ of rotation were acquired in $156 \mathrm{~ms}$. In addition a span time of $1.4 \mathrm{~s}$ was selected between scans in order to cover the full start of the foaming process. The fast acquisition of the projections ( $5 \mathrm{~ms}$ per projection) and the inherent viscosity of the mixture of polyol and isocyanate allowed minimizing the instability induced by the high rotation speed, consequently obtaining sharp reconstructions. The height of the rotation table was adjusted during time in order to compensate the sample expansion and consequently scanning the same zone of the sample during the whole experiment. Phase contrast projections were first converted to projected density maps [27], then the volumes were reconstructed using a Fourier-based GRIDEC 
algorithm [28]. Slices and 3D renderings of the comparatively scanned materials at several moments of the foaming process are shown in Fig. 1. This figure already shows a clear difference between the two materials, where nucleation is enhanced in the nanocomposite material.

(a)

(b)

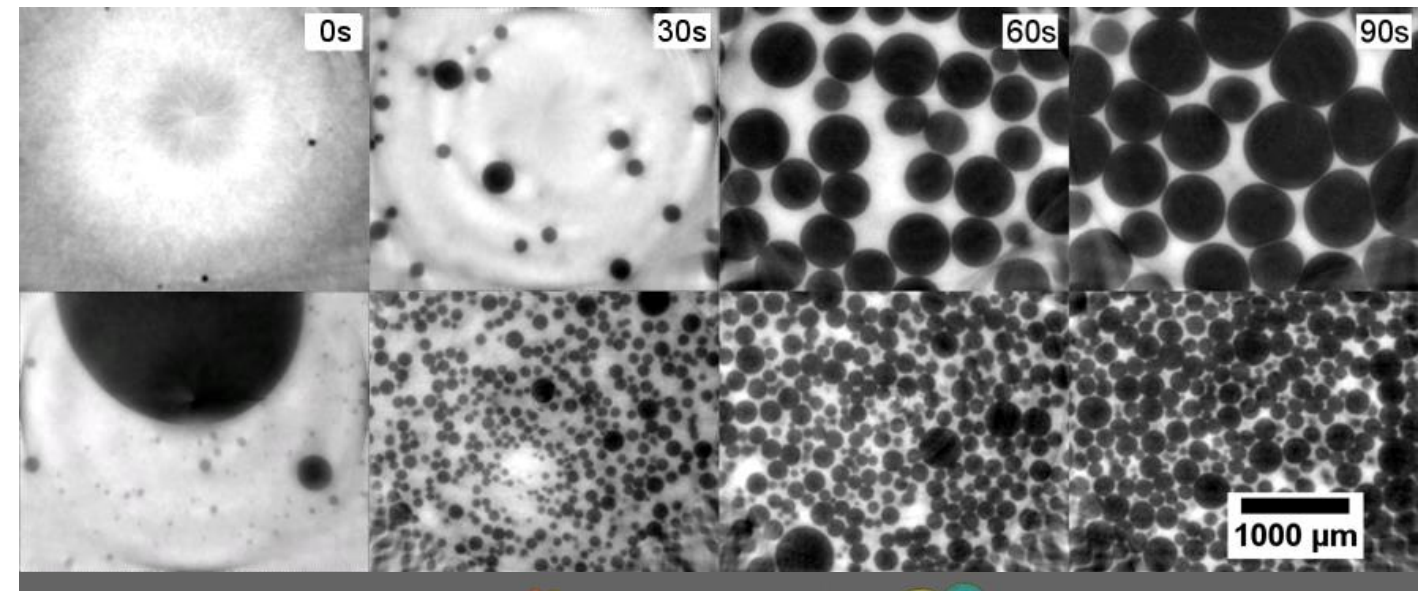

(c)

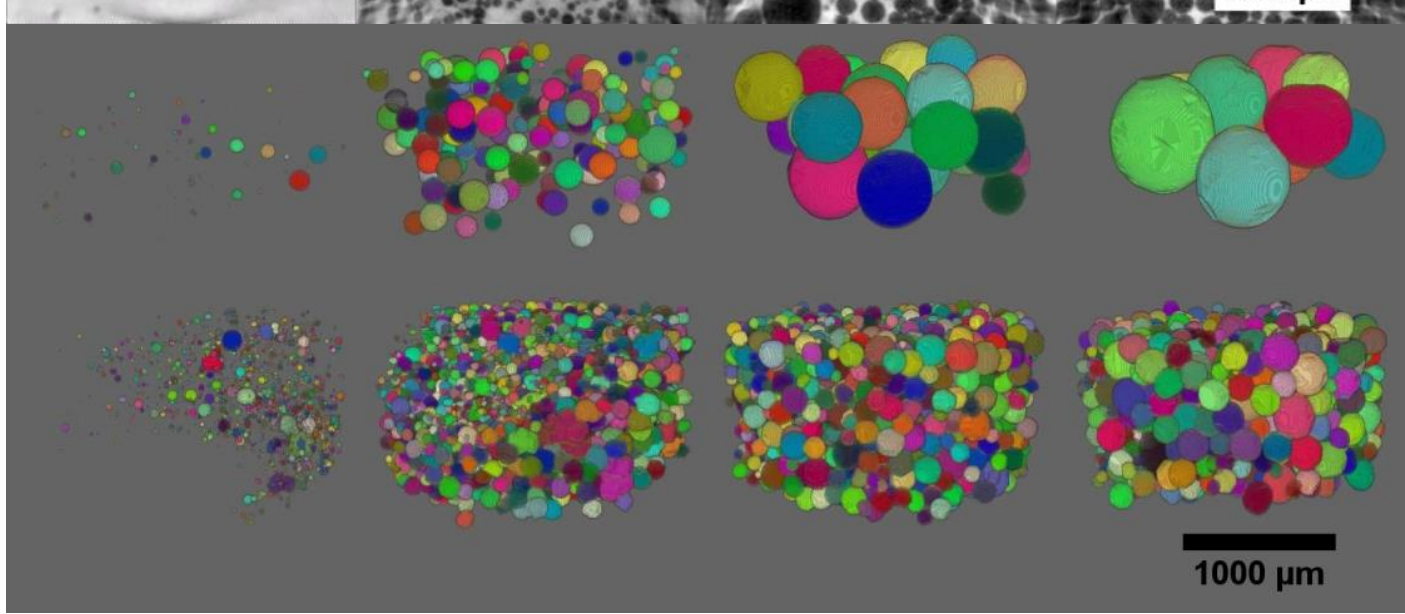

Fig. 1. Reconstructed slices for the two scanned systems at different times of the foaming process. Neat PU (a) and PU containing silica nanoparticles (b). 3D rendering of the analyzed cells at the same times in Neat PU (c) and PU containing silica nanoparticles (d).

\subsection{Image Analysis}

Data collected at TOMCAT were then quantitatively analyzed using the MorphoLibJ plugin library [29] of ImageJ/FIJI software tool [30, 31]. Firstly, an edge preserving filter was applied in order to facilitate the separation between gas and liquid. After that, the separation was carried out by using an adaptive binarization algorithm. As the cell walls are extremely thin in this kind of cellular polymers [32], a 3D distance transform watershed algorithm [33] was used in order to separate bubbles after binarization. As a result, we were able to study $3 \mathrm{D}$ descriptors of cellular material such as material density, bubble number (cell density) and its average size (cell size). This image analysis protocol 
was applied to 33 intercalated scans in the two materials covering approximately the initial 90s of the foaming process. 


\section{Results and Discussion}

Fig. 2 shows the foam relative density as a function of time. Relative density was calculated as the ratio between the volume occupied by the binarized liquid phase after watershed algorithm and the total volume. The inclusion of nanoparticles in the evolving liquid promotes a clear acceleration of the foaming process. A similar result was found when the foaming behavior of the same system was studied using in-situ FTIR. It was proved that this type of silica promotes a significant increase of the isocyanate consumption during the reaction increasing the reaction speed [26]. However final expansion factor is similar in both systems, reaching also similar final values of relative density ( 0.2). Observed incomplete expansion should be accomplished after reaching gel time of $145 \mathrm{~s}$.

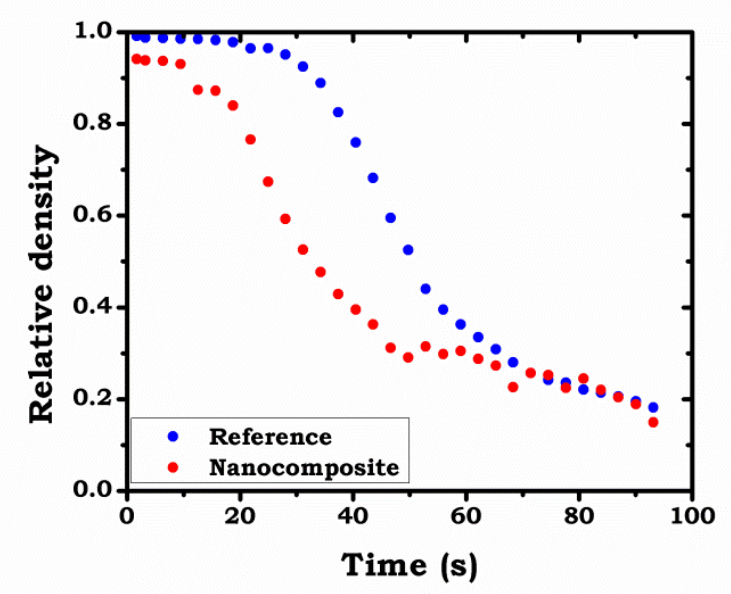

Fig. 2. In-situ relative density evolution for the two materials under study.

By analyzing the number of bubbles in each tomographic volume, cell nucleation density $\left(N_{0}\right)$ seems to become nearly constant after few seconds which is in congruence with nucleation theory (Fig. 3). In addition, it is clear that the inclusion of nanoparticles in the expanding liquid enhances the bubble nucleation density by two orders of magnitude (from $10^{4}$ to $10^{6}$ cells $\cdot \mathrm{cm}^{-3}$ ) due to the aforementioned free energy barrier reduction. In the literature, nucleation dynamics are usually modeled according to Arrhenius laws [2, $34,35]$. Thermodynamically, the reversible work of forming a germen of a new phase from a pre-existing metastable one comprises two contributions. The first one is associated with the cost of creating an interface and is therefore proportional to the surface area of the developing nuclei. The second one is related to the thermodynamic driving force tending to lower the overall free energy of the system by forming the stable phase, 
and is therefore proportional to the nuclei volume. The competition between these two contributions gives rise to a critically nuclei radius. This means that larger nuclei than the critical one grow spontaneously into the stable phase while smaller than the critical one shrink spontaneously and disappear into the metastable surroundings. Under these considerations, both homogeneous and heterogeneous nucleation rates can be estimated, at every instant, according to exponential equations from the classical nucleation theory as described in Eqns. 1-2. However, these models do not consider evolving conditions. In a real situation, it would be expected that the combination with other coupled not constant kinetics such as temperature, pressure or surface tension may finally lead to not constant nucleation rates. Nonetheless, in our particular case, we have demonstrated experimentally that the examined systems present constant nucleation rates of 1500 and $20000 \mathrm{~cm}^{-3} \cdot \mathrm{s}^{-1}$ for homogeneous and heterogeneous nucleation respectively (Fig. 3). Presence of nucleating particles has modified the nucleation rate of the original system in more than one order of magnitude.

$$
\begin{aligned}
& \left(d N_{0} / d t\right)^{\text {hom }}=C_{\text {hom }} f_{\text {hom }} \exp \left(-\frac{W}{k_{B} T}\right) \\
& \left(d N_{0} / d t\right)^{\text {het }}=C_{\text {het }} f_{\text {het }} \exp \left(-\frac{k W}{k_{B} T}\right)
\end{aligned}
$$

Where $C$ and $f$ are the concentration of gas molecules and the frequency factor of the gas molecules respectively, factors that in general depend weakly on temperature, referred for the homogeneous and heterogeneous nucleation systems. In addition, $W$ is the nucleation energy barrier or the reversible work needed to form a critical nucleus. The differences between the homogeneous and heterogeneous nucleation consider mainly an energy reduction factor, $k$, which depends on the geometry of the nucleating sites as expressed in the introduction and different pre-exponential factors $(C, f)$ considering the varying kinematic frequency factors in both situations. 


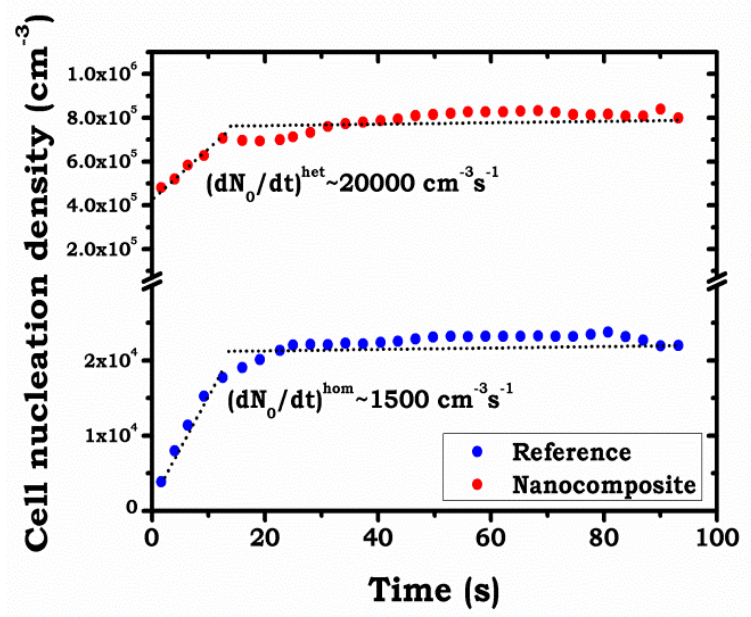

Fig. 3. Evolution of the three-dimensionally calculated cell density in the two scanned materials and the two calculated nucleation rates for both homogeneous and heterogeneous nucleation systems.

In our two scanned materials, it looks clear that cell nucleation follows a linear trend until approx. $15 \mathrm{~s}$, therefore validating the consideration of constant nucleation rate. In addition, after the first $15 \mathrm{~s}$ in which the nucleation takes place, it is remarkable that the cell nucleation becomes constant for both homogeneous and heterogeneous systems. Furthermore, the high number of nucleated bubbles at the start of the process in the nanocomposite system seems to indicate that the nucleation on this system starts during mixing process of two PU components.

Focusing on the evolution of the average size $(\phi)$ of the bubbles several authors proposed in the literature that the initial growth of spherical bubbles in an expanding liquid should fit well with $t^{1 / 2}[10,11,36]$ (Eqn. 3).

$\phi=6(3 / 4 \pi)^{1 / 3}(D t)^{1 / 2}$

where $D$ is the diffusion coefficient of the gas molecules within the polymer liquid phase.

In both systems (Fig. 4) cell size remains nearly constant during early instants of the process $(t<15 \mathrm{~s})$ while nucleation is taking place. Once the nucleation has been accomplished, the gas dissolved in the polymeric matrix starts to fill the bubbles at a nearly constant rate at this square-root scale, thus obtaining good linear fit of average bubble size versus $t^{1 / 2}$. However, this trend is only maintained until $\sim 65 \mathrm{~s}$ and $\sim 50 \mathrm{~s}$ for the neat PU and the formulation with nanoparticles respectively. At these times, both systems reach relative densities around 0.3 (Fig. 2). At this approximate value -so called wet-dry 
transition- cell shape changes from spherical to polyhedral shape [37]. In the wet regime (high relative density) the rounded bubbles are isolated in the liquid whereas in the dry regime (low relative density) the bubbles start touching and create three-dimensional polyhedral network. As a consequence, growth rate is slightly reduced after this reference value.

Therefore, the diffusion coefficient $(D)$ can be determined in each case from the observed trends (Fig. 4). In this sense, it has been demonstrated that, in addition to enhance the bubble nucleation, the inclusion of nanosilica particles into PU foam seems to decrease the gas diffusion through the liquid phase of the growing PU $\left(1.6 \cdot 10^{-7} v s .3 .5 \cdot 10^{-6} \mathrm{~cm}^{2} \cdot \mathrm{s}^{-}\right.$ ${ }^{1}$ for nanocomposite and neat PU respectively), commented broadly in the literature [3840]. In fact, diffusion coefficient of $\mathrm{CO}_{2}$ in different kinds of $\mathrm{PU}$ has been measured obtaining similar values using the high vacuum technique [41], by sorption weight comparison [42] or by gas permeability measurements [43]. Furthermore, when the cells start touching each other, in the wet-dry transition, the diffusion coefficient seems to decrease from $3.5 \cdot 10^{-6}$ to $3 \cdot 10^{-6} \mathrm{~cm}^{2} \cdot \mathrm{s}^{-1}$ and from $1.6 \cdot 10^{-7}$ to $4 \cdot 10^{-8} \mathrm{~cm}^{2} \cdot \mathrm{s}^{-1}$ for Neat and nanocomposite PU respectively. However, the change in the diffusion coefficient due to this transition is much lower than the one caused by the inclusion of nanoparticles into the evolving system. In this case, the decrease in growing rate (and thus in the diffusion coefficient) is caused by the absence of physical space that hinders the expansion of cells.

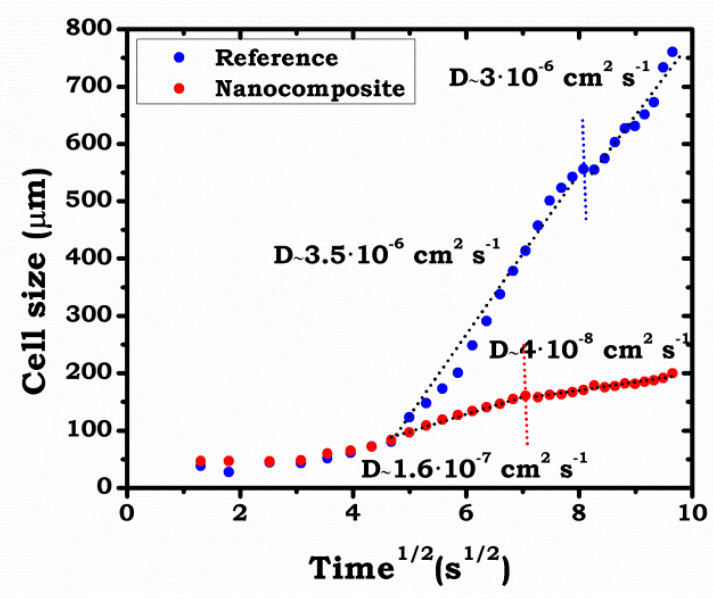


Fig. 4. Evolution of the average bubble size vs. the square root of time showing the commented regimes for the two scanned systems. The vertical lines cutting both plots are marking the wet-dry transition for both materials.

\section{Conclusions}

In conclusion, our 4D X-ray tomography study has provided us a unique insight to understand nucleation and growth processes the analyzed polyurethane formulations. Our analysis allowed us evaluating comparatively the evolution of critical characteristics such as foam density, cell nucleation density and cell size focusing on the early stages of the foaming process $(t<90 \mathrm{~s})$. We have found three differentiated regimes: nucleation, growth and wet-dry transition. Dynamics of these processes are highly influence by the presence of nanoparticles. Firstly, in the nucleation stage $(t<15 \mathrm{~s})$ the density slowly decreases, the cell nucleation density increases at constant rate and the cell size remains practically 
constant. In a subsequent stage, the growth takes place until the moment in which cells start touching each other (50-65s). In the analyzed remaining 75 seconds relative density drops up to nearly $70 \%$ of the final value, cell nucleation density remains constant and cell size increases following a $t^{1 / 2}$ rule in which the slope represents the diffusion coefficient of gas within the liquid polymer and obtaining similar values to those from literature for the PU-CO $\mathrm{CO}_{2}$ system. In this sense we have found that the inclusion of nanosilica particles decreases the diffusion coefficient. After the wet-dry transition (50s and 65 s for nanocomposite and neat PU respectively), both the density and the cell size evolution speed decrease whereas cell nucleation density still remains constant due to the reduction of physical space to grow. In addition, we have compared the obtained trends with those from nucleation and growth theories reaching valuable correlations. Up to our knowledge, this is the first work quantifying in situ and in three dimensions polymeric foams. In fact, in-situ studying the nucleation and growth of cells in 3D is of vital importance to evaluate experimentally these mechanisms and the reliability of the existing theoretical models. In this sense, this work has demonstrated that nucleation and growth are almost uncoupled mechanisms. In addition, both examined systems present almost constant nucleation and growth when the mechanisms take places, in good agreement with theoretical models.

\section{Acknowledgements}

Financial assistance from MINECO and FEDER program (MAT2015-69234-R) and the Junta de Castile and Leon (VA275P18) are gratefully acknowledged. Predoctoral contract of S. Perez-Tamarit by University of Valladolid (E-47-2015-0094701) and co-financed by Banco Santander is also acknowledged. Finally, we acknowledge the support from Paul Scherrer Institut (Villigen, Switzerland) to complete this work.

\section{References}


[1] S.F. Jones, G.M. Evans, K.P. BGalvin, Bubble nucleation from gas cavities - a review, Advances in Colloid and Interface Science 80(1) (1999) 27-50.

[2] M. Blander, J.L. Katz, Bubble nucleation in liquids, AIChe Journal 21(5) (1975) 833-848.

[3] F.F. Abraham, Homogeneous Nucleation Theory, Academic Press, New York, 1974.

[4] J.J. Feng, Prediction of bubble growth and size distribution in polymer foaming based on a new heterogeneous nucleation model, Journal of Rheology 48 (2004) 439-462.

[5] W.L. Ryan, E.A. Hemmingsen, Bubble formation in water at smooth hydrophobic surfaces, Journal of Colloid and Interfase Science 157(2) (1993) 312-317.

[6] J.S. Colton, N.P. Suh, The nucleation of microcellular thermoplastic foam with additives: Part I: Theoretical considerations, Polymer Engineering and Science 27(7) (1987) 485-492.

[7] L. Lee, C. Zeng, X. Cao, X. Han, J. Shen, G. Xu, Polymer nanocomposite foams, Composites Science and Technology 65(15-16) (2005) 2344-2363.

[8] M.M. Bernal, M.A. Lopez-Manchado, R. Verdejo, In situ Foaming Evolution of Flexible Polyurethane Foam Nanocomposites, Macromolecular Chemistry and Physics 212(9) (2011) 971-979.

[9] R. Verdejo, F.J. Tapiador, L. Helfen, M.M. Bernal, N. Bitinis, M.A. Lopez-Manchado, Fluid dynamics of evolving foams, Physical chemistry chemical physics : PCCP 11(46) (2009) 10860-6. [10] R.D. Pattel, Bubble growth in a viscous Newtonian liquid, Chemical Engineering Science 35(11) (1980) 2352-2356.

[11] M. Favelukis, R.J. Albalak, Bubble growth in viscous newtonian and non-newtonian liquids, The Chemical Engineering Journal 63(3) (1996) 149-155.

[12] D. Weaire, S. Hutzler, The physics of foams, First Edition ed.2001.

[13] I. Cantat, S. Cohen-Addad, F. Elias, F. Graner, R. Höhler, O. Pitois, F. Rouyer, A. Saint-Jalmes, Foams Structure and Dynamics, Oxford University Press2013.

[14] M.M. Bernal, S. Pardo-Alonso, E. Solórzano, M.A. Lopez-Machado, R. Verdejo, M.A. Rodriguez- Perez, Effect of carbon nanofillers on flexible polyurethane foaming from a chemical and physical perspective, RSC Advances 4(40) (2014) 20761-20768.

[15] S. Pardo-Alonso, E. Solórzano, S. Estravís, M.A. Rodriguez-Perez, J.A. de Saja, In situ evidence of the nanoparticle nucleating effect in polyurethane-nanoclay foamed systems, Soft Matter 8(44) (2012) 11262.

[16] E. Solórzano, S. PArdo-Alonso, J.A. de Saja, M.A. Rodriguez-Perez, X-ray radioscopy in-situ studies in thermoplastic polymer foams, Colloids and Surfaces A: Physicochemical and Engineering aspects 438 (2013) 167-173.

[17] E. Solórzano, S. Pardo-Alonso, J.A. de Saja, M.A. Rodríguez-Pérez, Study of aqueous foams evolution by means of X-ray radioscopy, Colloids and Surfaces A: Physicochemical and Engineering Aspects 438 (2013) 159-166.

[18] E. Solórzano, J. Pinto, S. Pardo, F. Garcia-Moreno, M.A. Rodriguez-Perez, Application of a microfocus X-ray imaging apparatus to the study of cellular polymers, Polymer Testing 32(2) (2013) 321-329.

[19] E. Maire, C. Le Bourlot, J. Adrien, A. Mortensen, R. Mokso, $20 \mathrm{~Hz}$ X-ray tomography during an in situ tensile test, International Journal of Fracture 200(1-2) (2016) 3-12.

[20] P.H. Kamm, F. García-Moreno, T.R. Neu, K. Heim, R. Mokso, J. Banhart, Fast Synchrotron XRay Tomography of Dynamic Processes in Liquid Aluminium Alloy Foam Advanced Engineering Materials 19(11) (2017) 1600550.

[21] G. Lovric, R. Mokso, C.M. Schleputz, M. Stampanoni, A multi-purpose imaging endstation for high-resolution micrometer-scaled sub-second tomography, Physica medica : PM : an international journal devoted to the applications of physics to medicine and biology : official journal of the Italian Association of Biomedical Physics 32(12) (2016) 1771-1778.

[22] T.J. Davis, D. Gao, T.E. Gureyev, A.W. Stevenson, S.W. Wilkins, Phase-contrast imaging of weakly absorbing materials using hard X-rays, Nature 373 (1995) 595-598.

[23] O. Betz, U. Wegst, D. Weide, M. Heethof, L. Helfen, W.-K. Lee, P. Cloetens, Imaging applications of synchrotron X-ray phase-contrast microtomography in biological morphology 
and biomaterials science. I. General aspects of the technique and its advantages in the analysis of millimetre-sized arthropod structure, Journal of Microscopy 227 (2007) 51-71.

[24] A. Momose, A. Fujii, H. Kadowaki, H. Jirai, Three-Dimensional Observation of Polymer Blend by X-ray Phase Tomography, Macromolecules 38 (2005) 7197-7200.

[25] S. Estravís, J. Tirado-Mediavilla, M. Santiago-Calvo, J.L. Ruiz-Herrero, F. Villafañe, M.Á. Rodríguez-Pérez, Rigid polyurethane foams with infused nanoclays: Relationship between cellular structure and thermal conductivity, European Polymer Journal 80 (2016) 1-15.

[26] M. Santiago-Calvo, J. Tirado-Mediavilla, J.L. Ruiz-Herrero, M.Á. Rodríguez-Pérez, F. Villafañe, The effects of functional nanofillers on the reaction kinetics, microstructure, thermal and mechanical properties of water blown rigid polyurethane foams, Polymer 150 (2018) 138-149.

[27] D. Paganing, S.C. Mayo, T.E. Gureyev, P.R. Miller, S.W. Wilkins, Simultaneous phase and amplitude extraction from a single defocused image of a homogeneous object, Journal of Microscopy 206(1) (2002) 33-40.

[28] F. Marone, C. Hintermüller, R. Geus, M. Stampanoni, Towards real-time tomography fast reconstruction algorithms and GPU implementation, IEEE Nuclear Science Symposium Conference Proceedings 1-9 (2009).

[29] D. Legland, I. Arganda-Carreras, P. Andrey, MorphoLibJ: integrated library and plugins for mathematical morphology with ImageJ, Bioinformatics 32(22) (2016) 3532-3534.

[30] M.D. Abràmoff, P.J. Magalhaes, J. Sunanda, Image Processing with ImageJ, Biophotonics International 11(7) (2004) 36-42.

[31] C.A. Schneider, W.S. Rasband, K.W. Eliceiri, NIH Image to ImageJ: 25 years of image analysis, Nature Methods 9(7) (2012) 671-675.

[32] S. Pardo-Alonso, E. Solórzano, L. Brabant, P. Vanderniepen, M. Dierick, L. Van Hoorebeke, M.A. Rodriguez- Perez, 3D Analysis of the progressive modification of the cellular architecture in polyurethane nanocomposite foams via X-ray microtomography, European Polymer Journal 49 (2013) 999-1006.

[33] L. Vincent, P. Soille, Watersheeds in digital spaces: An efficient algorithm based on immersion simulations, IEEE Transactions on Pattern Analysis and Machine Intelligence 13(6) (1991) 583-598.

[34] J.S. Colton, The Nucleation of Microcellular Foams in Semicrystalline Thermoplastics, Materials and Manufacturing Processes 4(2) (1989) 253-262.

[35] J.S. Colton, N.P. Suh, The Nucleation of Microcellular Thermoplastic Foam with Additives: Part II: Experimental Results and Discussion, Polymer Engineering and Science 27 (1987) 493499.

[36] M. Amon, C.D. Denson, A study of the dynamics of foam growth: Analysis of the growth of closely spaced spherical bubbles, Polymer Engineering and Science 24(13) (1984) 1026-1034.

[37] Y. Furuta, N. Oikawa, R. Kurita, Close relationship between a dry-wet transition and a bubble rearrangement in two-dimensional foam, Scientific reports 6 (2016) 37506.

[38] E. Rio, W. Drenckhan, A. Salonen, D. Langevin, Unusually stable liquid foams, Adv Colloid Interface Sci 205 (2014) 74-86.

[39] A. Stocco, E. Rio, B.P. Binks, D. Langevin, Aqueous foams stabilized solely by particles, Soft Matter 7(4) (2011) 1260.

[40] B.P. Binks, R. Murakami, Phase inversion of particle-stabilized materials from foams to dry water, Nature materials 5(11) (2006) 865-9.

[41] J.S. McBride, T.A. Massaro, S.L. Cooper, Diffusion of gases through polyurethane block polymers, Journal of Applied Polymer Science 23 (1979) 201-214.

[42] D. Hu, L. Yan, T. Liu, Z. Xu, L. Zhao, Solubility and diffusion behavior of compressed CO2 in polyurethane oligomer, Journal of Applied Polymer Science 135 (2018) 47100.

[43] N. Cao, M. Pegoraro, F. Bianchi, L. Di Landro, L. Zanderighi, Gas transport properties of polycarbonate-polyurethane membranes, Journal of Applied Polymer Science 48(10) (1993) 1831-1842. 
